\title{
Spin squeezing via one-axis twisting with coherent light
}

\section{$\operatorname{AUTHOR}(\mathrm{S})$ :}

Takeuchi, M; Ichihara, S; Takano, T; Kumakura, M; Yabuzaki, T; Takahashi, Y

\section{CITATION:}

Takeuchi, M ... [et al]. Spin squeezing via one-axis twisting with coherent light. PHYSICAL REVIEW LETTERS 2005, 94(2): 023003.

\section{ISSUE DATE:}

2005-01-21

URL:

http://hdl.handle.net/2433/49886

RIGHT:

Copyright 2005 American Physical Society 


\title{
Spin Squeezing via One-Axis Twisting with Coherent Light
}

\author{
M. Takeuchi, ${ }^{1}$ S. Ichihara, ${ }^{1}$ T. Takano, ${ }^{1}$ M. Kumakura, ${ }^{1,2}$ T. Yabuzaki, ${ }^{1}$ and Y. Takahashi ${ }^{1,2}$ \\ ${ }^{1}$ Department of Physics, Graduate School of Science, Kyoto University, Kyoto 606-8502, Japan \\ ${ }^{2}$ CREST, JST, 4-1-8 Honcho Kawaguchi, Saitama 332-0012, Japan
}

(Received 17 June 2004; published 18 January 2005)

\begin{abstract}
We propose a new method of spin squeezing of atomic spin, based on the interactions between atoms and off-resonant light which are known as paramagnetic Faraday rotation and the fictitious magnetic field of light. Since the projection process, squeezed light, or special interactions among the atoms are not required in this method, it can be widely applied to many systems. The attainable range of the squeezing parameter is $\zeta \geqslant S^{-2 / 5}$, where $S$ is the total spin, which is limited by additional fluctuations imposed by coherent light and the spherical nature of the spin distribution.
\end{abstract}

DOI: 10.1103/PhysRevLett.94.023003

PACS numbers: 32.80.-t, 03.67.Mn, 42.50.Lc

The squeezed spin state (SSS) is one of the nonclassical states in the collective spin system. In the SSS, the quantum uncertainty of the spins along an axis orthogonal to the mean spin vector $\left\langle\Delta \tilde{S}_{\perp}^{2}\right\rangle$ is suppressed below the standard quantum limit (SQL) such as $\left\langle\Delta \tilde{S}_{\perp}^{2}\right\rangle<|\langle\tilde{\mathbf{S}}\rangle| / 2$, where $\langle\tilde{\mathbf{S}}\rangle$ is the mean spin vector, due to an entanglement formation among the individual spins. The degree of the squeezing is usually evaluated by the squeezing parameter $\zeta \equiv$ $2\left\langle\Delta \tilde{S}_{\perp}^{2}\right\rangle /|\langle\tilde{\mathbf{S}}\rangle|$, in terms of the variance to average ratio [1].

For the last several years, the SSS has been extremely interesting not only for its precision measurement of the spin component [2-4], but also for its application to quantum information [5-7]. There have been many proposals and experiments to realize the spin squeezing of atoms. They can be put into three categories as follows: (i) Quantum nondemolition (QND) measurement of spin via paramagnetic Faraday rotation and spin squeezing by quantum projection [5,8-13]. The QND measurement has already been performed by some groups for the electronic ground states of the atom $[5,11,13]$, and the squeezed parameter has reached about $\zeta \sim 0.7$ for $S \sim 4 \times 10^{7}$ [11] and $\zeta \sim 0.1$ for $S \sim 10^{11}$ [13]. Since the projection causes the squeezing in this method, the degree of the squeezing will finally be determined by the performance of the detector. (ii) Quantum-state transfer from squeezed light to spin [6,7,14-17]. One type is based on the complete absorption of the squeezed vacuum, and has been experimentally demonstrated for the electronic excited states of the atom $\left(\zeta \sim 0.97\right.$ for $\left.S \sim 5 \times 10^{7}\right)$ [14]. Another type is based on the stimulated Raman adiabatic passage $[6,7,16,17]$. Since the squeezed light is the source of spin squeezing in these methods, the degree of the squeezing will finally be determined by the quality of the squeezed light. (iii) Special systems to induce nonlinear interactions among the individual spins such as BoseEinstein condensates [18-20], cold atoms in optical lattice [21], and atoms in the optical cavity [22-24]. They are not easy to prepare and difficult to operate after squeezed.
In this Letter, we propose a new method to realize the spin squeezing, which cannot be put into any of the three categories. Our method does not rely on the projection by the measurement, the use of squeezed light, and the specialities of the systems. Instead, the new method requires only a coherent light pulse and a few linear optics, so it can be widely applied to many systems. It should be noted that a recent report by Hammerer et al. [25] includes another proposal of an unconditional spin squeezing with coherent light.

Our method is based on the interaction between atoms and off-resonant light, whose interaction Hamiltonian takes the form [9]

$$
H=\alpha J_{z} S_{z}
$$

where $\alpha$ is a real constant, and the $z$ axis is set parallel to the wave vector of the light. $\mathbf{S}$ is the summation over the individual spin, which obeys the usual commutation relation of angular momenta $\left[S_{i}, S_{j}\right]=i \varepsilon_{i j k} S_{k} . \mathbf{J}$ is the quantum-mechanical Stokes vector of light, which also obeys the usual commutation relation of angular momenta $\left[J_{i}, J_{j}\right]=i \varepsilon_{i j k} J_{k}$. For a light pulse with the duration $T$ propagating in free space, $\mathbf{J}$ can be written as $J_{x} \equiv \frac{1}{2} \times$ $\int_{0}^{T}\left(a_{+}^{\dagger} a_{-}+a_{-}^{\dagger} a_{+}\right) d t, \quad J_{y} \equiv \frac{1}{2 i} \int_{0}^{T}\left(a_{+}^{\dagger} a_{-}-a_{-}^{\dagger} a_{+}\right) d t$, $J_{z} \equiv \frac{1}{2} \int_{0}^{T}\left(a_{+}^{\dagger} a_{+}-a_{-}^{\dagger} a_{-}\right) d t$, where $a_{ \pm}$is the annihilation operators of $\sigma_{ \pm}$circular polarization mode, respectively [26]. The interaction of Eq. (1) represents the addition of the phase difference for $\sigma_{ \pm}$light, which causes the rotation of the polarization plane for linear polarization at the angular frequency $\alpha S_{z} / 2$, known as paramagnetic Faraday rotation. It also represents the spin rotation around the $z$ axis at the angular frequency $\alpha J_{z}$, known as the fictitious magnetic field of light [27]. If we are able to apply a light pulse whose $J_{z}$ is proportional to $S_{z}$ as a fictitious magnetic field, the collective spin will nonlinearly rotate at angular frequencies proportional to $S_{z}$, whose evolution will be similar to one-axis twisting [1]. This is the basic idea of our proposal. 


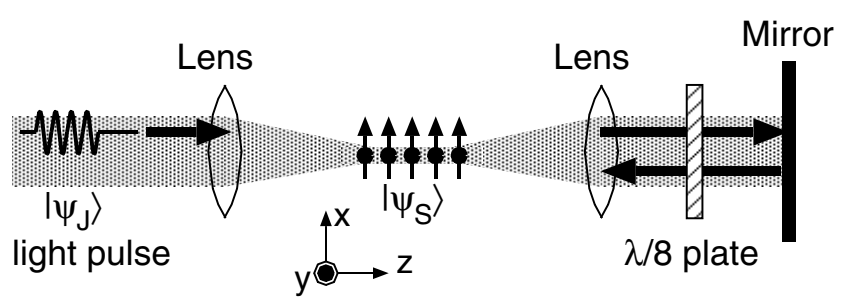

FIG. 1. System of our proposal. A linearly polarized light pulse passes through an atomic ensemble and the polarization plane is rotated. The rotation angle is proportional to $S_{z}$ and converts to the circular polarization components after passing through the $\lambda / 8$ plate twice. When the pulse passes through the atomic ensemble again, the pulse induces a nonlinear rotation to the atomic ensemble around the $z$ axis as a fictitious magnetic field. See text for details.

To design such an interaction, we propose the system illustrated in Fig. 1. Initially, a light pulse $\left|\psi_{J}\right\rangle$ is linearly polarized along the $x$ axis and contains $2 J(\gg 1)$ photons as an average. Atoms $\left|\psi_{S}\right\rangle$ are spin polarized along the $x$ axis and contains total spin $S$. The light is weakly focused to match the atomic ensemble [26]. The averages of the Stokes components are then $\left\langle J_{x}\right\rangle=J,\left\langle J_{y}\right\rangle=\left\langle J_{z}\right\rangle=0$, and the averages of the collective spin components are $\left\langle S_{x}\right\rangle=S,\left\langle S_{y}\right\rangle=\left\langle S_{z}\right\rangle=0$. Since the light pulse is a strong coherent state, we can approximate the commutation relation as $\left[J_{y}, J_{z}\right]=i J[26]$. First, a light pulse passes through the atoms and the polarization plane is then rotated. We call it "the first interaction," whose interaction time is labeled as $t_{1}$. The Stokes vector becomes $\mathbf{J}^{(\mathrm{FI})}=$ $e^{i t_{1} H} \mathbf{J} e^{-i t_{1} H}$, whose $y$ component is approximately written as $J_{y}^{(\mathrm{FI})} \simeq J_{y}+\alpha t_{1} J S_{z}$, for $\alpha t_{1} S_{z} \ll 1$. Since the average of $J_{y}^{(\mathrm{FI})}$ becomes $\left\langle\psi_{J}\left|J_{y}^{(\mathrm{FI})}\right| \psi_{J}\right\rangle=\alpha t_{1} J S_{z}$, we can say that the information of $S_{z}$ is copied and held on $J_{y}^{(\mathrm{FI})}$ as a Faraday rotation angle. We note that $S_{z}$ is conserved because the interaction of Eq. (1) satisfies the backaction evasion (BAE) condition of $\left[S_{z}, H\right]=0$. Second, the pulse passes through twice the $\lambda / 8$ wave plate by the totally retroreflecting mirror. As a result, the $\lambda / 4$ phase difference is induced between the two orthogonal modes of linear polarization. We call it "the local operation" for the light. The Stokes vector becomes $\mathbf{J}^{(\mathrm{LO})}=e^{i(\pi / 2) J_{x}} \mathbf{J}^{(\mathrm{FI})} e^{-i(\pi / 2) J_{x}}$, whose $z$ component is $J_{z}^{(\mathrm{LO})}=J_{y}^{(\mathrm{FI})}$. We can say that the information of $S_{z}$ is shifted from $J_{y}^{(\mathrm{FI})}$ to $J_{z}^{(\mathrm{LO})}$, converting the angle of the polarization plane to the photon number difference of the $\sigma_{ \pm}$modes. Thus, the required light is achieved whose $J_{z}$ is approximately proportional to $S_{z}$. Finally, the pulse passes through the atomic ensemble again. We call it "the second interaction," whose interaction time is labeled as $t_{2}$. The interaction Hamiltonian of the second interaction can be roughly written as $H^{(\mathrm{SI})} \sim$ $\alpha J_{z}^{(\mathrm{LO})} S_{z} \propto S_{z}^{2}$, which takes a form similar to the one-axis twisting Hamiltonian $\chi S_{z}^{2}$ [1]. Thus, we can expect that the spin state becomes the SSS after the second interaction.

Next, we derive the density operator of the spin after the second interaction to calculate the properties of the spin state obtained by this method. The initial density operator of the whole system can be written as $\rho_{S J} \equiv \rho_{S} \otimes$ $\left|\psi_{J}\right\rangle\left\langle\psi_{J}\right|$, where $\rho_{S}=\left|\psi_{S}\right\rangle\left\langle\psi_{S}\right|$. After the second interaction, it becomes $\tilde{\rho}_{S J} \equiv U \rho_{S J} U^{\dagger}$ where $U=$ $e^{-i t_{2} H} e^{-i(\pi / 2) J_{x}} e^{-i t_{1} H}$. The reduced density operator representing the spin state after the second interaction $\tilde{\rho}_{S}$ can be written as $\tilde{\rho}_{S}=\operatorname{Tr}_{J}\left(\tilde{\rho}_{S J}\right)$, where $\operatorname{Tr}_{J}$ is the partial trace for the light. For convenience, we consider the set of eigenstates for $\mathbf{S}^{2}$ and $S_{z}$, say $|S, M\rangle$, where $\mathbf{S}^{2}|S, M\rangle=S(S+$ 1) $|S, M\rangle$ and $S_{z}|S, M\rangle=M|S, M\rangle$. The matrix elements take the form

$$
\begin{gathered}
\left\langle S, M\left|\tilde{\rho}_{S}\right| S, M^{\prime}\right\rangle=\sigma_{M M^{\prime}}\left\langle S, M\left|\rho_{S}\right| S, M^{\prime}\right\rangle, \\
\sigma_{M M^{\prime}}=e^{-\mu^{\prime}\left(M-M^{\prime}\right)^{2} / 2} e^{-i \mu\left(M^{2}-M^{\prime 2}\right) / 2},
\end{gathered}
$$

where we have set $\mu \equiv\left(\alpha t_{1}\right)\left(\alpha t_{2}\right) J$ and $\mu^{\prime} \equiv\left[\left(\alpha t_{1}\right)^{2}+\right.$ $\left.\left(\alpha t_{2}\right)^{2}\right] J / 2$. If $t_{1}=t_{2}$ then $\mu=\mu^{\prime}$. Since the atoms $\left|\psi_{S}\right\rangle$ are polarized along the $x$ axis, the matrix elements of $\rho_{S}$ can be written as

$$
\left\langle S, M\left|\rho_{S}\right| S, M^{\prime}\right\rangle=\frac{1}{2^{2 S}}\left(\begin{array}{c}
2 S \\
S+M
\end{array}\right)^{1 / 2}\left(\begin{array}{c}
2 S \\
S+M^{\prime}
\end{array}\right)^{1 / 2} .
$$

In the following discussions, we use the expressions of Eqs. (3) and (4). We note that the ideal one-axis twisted state [1] corresponds to the case of $\mu^{\prime}=0$.

To know how uncertainties evolve, we calculate the quasiprobability distributions (QPD), which is defined as $Q(\theta, \phi)=\left\langle\theta, \phi\left|\tilde{\rho}_{S}\right| \theta, \phi\right\rangle$, where $|\theta, \phi\rangle \equiv$ $e^{-i \phi S_{z}} e^{-i \theta S_{y}}|S, S\rangle$ is a spin state polarized along the direction whose polar and azimuth angles are $\theta$ and $\phi$, respectively [1]. The results of the calculations in the case of $S=20$ are shown in Fig. 2 for the initial spin state (a), the spin state after the first interaction (b), and that after the second (c). The initial spin state is isotropically distributed along the $x$ direction as is shown in Fig. 2(a). After the first
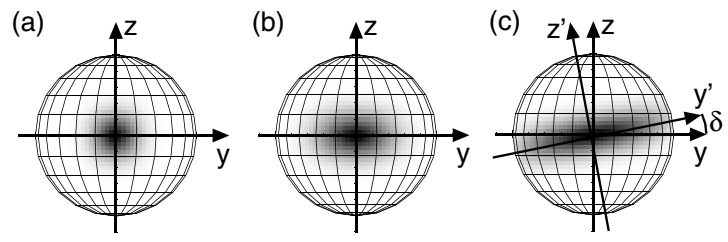

FIG. 2. State evolutions expressed as the quasiprobability distribution for $S=20$. The value of QPD for the $(\theta, \phi)$ direction is represented by the gray scale on the unit sphere, which is normalized by the maximum value. (a) The initial spin state. (b) The spin state after the first interaction, where we have set $\left(\alpha t_{1}\right)^{2} J / 2=0.1$ and $t_{2}=0$; in other words, $\mu=0$ and $\mu^{\prime}=$ 0.1 . (c) The spin state after the second interaction, where we have set $\left(\alpha t_{1}\right)^{2} J / 2=\left(\alpha t_{2}\right)^{2} J / 2=0.1$; in other words, $\mu=$ $\mu^{\prime}=0.2$. The spin squeezing is realized along the $z^{\prime}$ axis. 
interaction, the distribution is a little broadened along the $y$ direction, as Fig. 2(b) indicates. This is explained by additional fluctuation imposed by coherent light. In fact, the $y$ component after the first interaction is approximately written as $S_{y}^{(\mathrm{FI})}=e^{i t_{1} H} S_{y} e^{-i t_{1} H} \simeq S_{y}+\alpha t_{1} J_{z} S_{x}$ for $\alpha t_{1} J_{z} \ll$ 1. Since $S_{z}$ is the BAE variable, the distribution along the $z$ direction does not change at all. After the second interaction, the distribution looks twisted around the $z$ axis and squeezed along the $z^{\prime}$ axis, as Fig. 2(c) indicates. Although not clear from the figure, the distribution is also broadened along the $y$ axis as in the case after the first interaction. In fact, the $y$ component after the second interaction is roughly written as $\tilde{S}_{y} \sim e^{i t_{2} H^{(\mathrm{SI})}} S_{y}^{(\mathrm{FI})} e^{-i t_{2} H^{(\mathrm{SI})}} \sim S_{y}+$ $\mu S_{z} S_{x}+\left(\alpha t_{1} J_{z}+\alpha t_{2} J_{y}\right) S_{x}$. By these additional fluctuations imposed by coherent light, the spin state after the second interaction is different from the ideal one-axis twisted state [1]. The additional fluctuations would be reduced by the use of a polarization squeezed light pulse whose squeezed component is $t_{1} J_{z}+t_{2} J_{y}$, approaching the ideal one-axis twisting interaction of $\mu^{\prime} \rightarrow 0$. We mention that the additional fluctuations by light in the method of Ref. [25] are imposed on both the $y$ and the $z$ components, while the $z$ component is squeezed. Therefore, the squeezing parameter does not become small in that scheme.

From Eqs. (3) and (4), we can derive the averages and variances of the spin components. The averages can be calculated as $\left\langle\tilde{S}_{x}\right\rangle=S e^{-\mu^{\prime} / 2} \cos ^{2 S-1}(\mu / 2)$ and $\left\langle\tilde{S}_{y}\right\rangle=$ $\left\langle\tilde{S}_{z}\right\rangle=0$, where $\tilde{\mathbf{S}}$ represents the spin operator after the second interaction. They indicate that the orientation of the mean spin vector remains the $(\pi / 2,0)$ direction or the $x$ axis, as is shown in Fig. 2(c). To characterize an elliptical distribution around the $x$ axis, we define the minor and major axes, say $z^{\prime}$ and $y^{\prime}$, respectively, as is shown in Fig. 2(c), so that the variances of those components $\left\langle\Delta \tilde{S}_{z^{\prime}}^{2}\right\rangle$ and $\left\langle\Delta \tilde{S}_{y^{\prime}}^{2}\right\rangle$ give the minimum and maximum, respectively, on the $y-z$ plane. The variances can be calculated as $\left\langle\Delta \tilde{S}_{x}^{2}\right\rangle=S^{2}-\left\langle\tilde{S}_{x}\right\rangle^{2}-S(S-1 / 2) A / 2$ and

$$
\left\langle\Delta \tilde{S}_{\substack{y^{\prime} \\ z^{\prime}}}^{2}\right\rangle=\frac{S}{2}+\frac{S}{2} \frac{S-1 / 2}{2}\left(A \pm \sqrt{A^{2}+B^{2}}\right),
$$

where we have set $A=1-e^{-2 \mu^{\prime}} \cos ^{2 S-2} \mu$ and $B=$ $4 e^{-\mu^{\prime} / 2} \sin (\mu / 2) \cos ^{2 S-2}(\mu / 2)$. We can also calculate $\delta$, which is an angle between the directions of the $z^{\prime}$ and $z$ axes or the $y^{\prime}$ and $y$ axes, as is shown in Fig. 2(c), and obtain $\delta=\arctan (B / A) / 2$. For $S \gg 1$ and $S^{-1} \ll$ $\mu \sim \mu^{\prime} \ll S^{-1 / 2}$, we find the approximate value of the variance of the $z^{\prime}$ component

$$
\left\langle\Delta \tilde{S}_{z^{\prime}}^{2}\right\rangle \simeq \frac{S}{2}\left(\frac{\gamma^{\prime}}{\gamma^{2}+\gamma^{\prime}}+\frac{2}{3} \beta^{2}\right),
$$

where we have set $\gamma=S \mu / 2, \gamma^{\prime}=S \mu^{\prime} / 2$, and $\beta=$ $S \mu^{2} / 4$. Also we find $\left\langle\tilde{S}_{x}\right\rangle \simeq S(1-\beta)$.
To examine the dependence on the interaction strength $\alpha t_{1}, \alpha t_{2}$ and the input photon number $2 J$, we plot the variances of the $y^{\prime}$ components and the $z^{\prime}$ components as a function of $\mu\left(=\mu^{\prime}\right)$ in Fig. 3(a). We also plot the approximate value for the $z^{\prime}$ components written as Eq. (6). It is clearly known that the variance of the $z^{\prime}$ component is reduced for small $\mu$, minimized at an optimal value of $\mu$, and becomes large for large $\mu$. It means that too strong an interaction or too large a photon number deteriorates the squeezing. This is explained by the spherical nature of the spin distribution, and, in fact, the variance of the $y^{\prime}$ component is almost saturated at the largest value of $S^{2} / 2$ for large $\mu$, which was entirely ignored in the analysis in Ref. [25]. As a typical value of $\mu$, we introduce $\mu_{\text {half }}$ as the value of $\mu$ to attain $\left\langle\Delta \tilde{S}_{z^{\prime}}^{2}\right\rangle=S / 4$, the half variance of the SQL. We also introduce $\mu_{\min }$ as the value to attain the minimum of $\left\langle\Delta \tilde{S}_{z^{\prime}}^{2}\right\rangle$. We plot the numerical solutions of $\mu_{\text {half }}$ and $\mu_{\min }$ in Fig. 3(b) for the case of $\mu=$ $\mu^{\prime}$. One can see that both $\mu_{\text {half }}$ and $\mu_{\text {min }}$ become small as $S$ increases but they obey different power laws. From Eq. (6), we find $\mu_{\text {half }} \simeq 2 S^{-1}$ and $\mu_{\min } \simeq 2(3 / 2)^{1 / 5} S^{-3 / 5}$. We show these approximate solutions in Fig. 3(b), which are in good agreement with the numerical ones. We also find that the squeezing parameter at $\mu=\mu^{\prime}=\mu_{\min }$ becomes $\zeta_{\min } \simeq$ $(2 / 3)^{1 / 5} S^{-2 / 5}$. We note that it is slightly worse than $(1 / 3)^{1 / 3} S^{-2 / 3}$, which is the squeezing parameter for the ideal one-axis twisting, due to the additional fluctuations imposed by coherent light, as is mentioned above.

Finally, we discuss the feasibility of our method. In the following, we consider the case that the shape of the light pulse is a square wave with its peak power $P$ and pulse duration $T$. As in Ref. [26], we assume $\Delta \gg \Gamma, \Omega \ll \Delta$, and $r T \ll(S \mu)^{-1} \ll 1$, where $\Delta$ represents the detuning from the resonance frequency, $\Gamma$ the full natural linewidth at half maximum of the transition, $\Omega$ the Rabi frequency, (a)

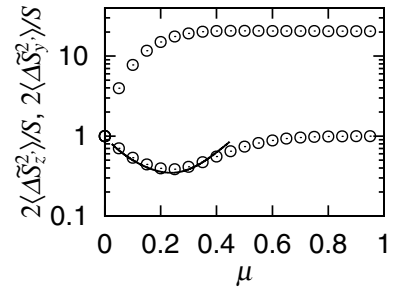

(b)

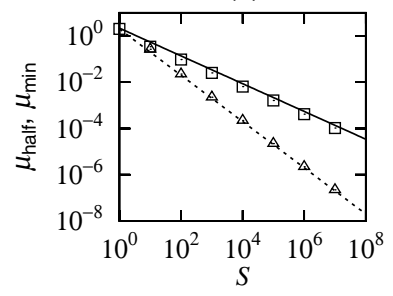

FIG. 3. (a) Variances of the $z^{\prime}$ (circle below 1) and the $y^{\prime}$ (circle above 1) components for $S=20$ as a function of $\mu$ ( $\mu_{\text {half }}=$ $0.117, \mu_{\min }=0.236$ ). They are normalized as $2\left\langle\Delta \tilde{S}_{z^{\prime}}^{2}\right\rangle / S$ and $2\left\langle\Delta \tilde{S}_{y^{\prime}}\right\rangle / S$, respectively. We also plot the approximate value of $2\left\langle\Delta S_{z^{\prime}}^{2}\right\rangle / S$ (solid line). (b) Values of $\mu_{\text {half }}$ (triangle) and $\mu_{\text {min }}$ (square) as a function of $S$, which are the required values of $\mu$ to obtain the half variance of the SQL and the attainable minimum variance, respectively. We also plot the approximate solutions of $\mu_{\text {half }}$ (dashed line) and $\mu_{\min }$ (solid line). To obtain both (a) and (b), we have assumed $\mu=\mu^{\prime}$. 
and $r$ the photon scattering rate [28]. After some calculations, we obtain $\mu=\mu^{\prime}=r T \sigma_{0} /\left(2 \pi w^{2}\right)$, where $w$ represents the beam waist and $\sigma_{0}$ the photon-absorption cross section of an atom, which can be written as $\sigma_{0}=$ $3 \lambda_{0}^{2} /(2 \pi)$ with the resonance wavelength $\lambda_{0}$. We note that $\Omega, r$, and $\mu$ are exactly the same as $2 g \sqrt{2 N_{p} /(c T)}$, $4 \varepsilon_{a} / T$, and $\kappa^{2} / N_{a}$ in Ref. [26], respectively. The condition to obtain $\mu \geq \mu_{\text {half }}$, or $\zeta \leq 1 / 2$, can be rewritten as $d_{0} \geq$ $8(r T)^{-1}$, where $d_{0}=2 S \sigma_{0} /\left(\pi w^{2}\right)$ is the optical depth. We also note that this condition is the same as $\kappa \geq \sqrt{2}$, similar to that of the QND measurement [26]. Such a condition has been satisfied in several systems, such as atoms in a cell [5], laser cooled and trapped atom, and so on. The feasibility of our scheme also comes from the simple experimental setup depicted in Fig. 1, which is also the great advantage over another scheme in Ref. [25]. This suggests that efficient squeezing can be realized by the current technologies.

As one ideal example, we consider ytterbium atoms $\left({ }^{171} \mathrm{Yb}\right)$ in an optical trap $[29,30]$, which contains $S=4 \times$ $10^{6}$. The atom collision and the precession due to the stray magnetic field, which causes the transverse relaxation, are well suppressed because it is an ultracold fermion and has only a nuclear spin $1 / 2$ whose gyromagnetic ratio is about three orders smaller than paramagnetic atoms like alkali metal. From the parameters given in Ref. [30], $w=3 \mu \mathrm{m}$, $\lambda_{0}=399 \mathrm{~nm}$, and $\Gamma=2 \pi \times 29 \mathrm{MHz}$, the light pulse of $\mu=5.4 \times 10^{-6}$, for example, is obtained by setting $\Delta=$ $2 \pi \times 24 \mathrm{GHz}, P=17 \mathrm{nW}, T=0.24 \mathrm{~ms}$, which satisfies the assumptions $\Delta \gg \Gamma, \Omega=2 \pi \times 21 \mathrm{MHz} \ll \Delta, r T=$ $4.0 \times 10^{-3} \ll(S \mu)^{-1}=4.6 \times 10^{-2} \ll 1$, and $J=4.0 \times$ $10^{6} \gg 1$. In this case the squeezing parameter becomes $\zeta=0.08$. We note that the decay constant of the atom number in the optical trap is about $4 s$ [31], which is so much longer than the pulse duration $T$ that we can treat the total spin $S$ as a constant. We also note that the length of the atom distribution should be adjusted to $L \sim 70 \mu \mathrm{m}$ to satisfy the condition of $\pi w^{2} /\left(\lambda_{0} L\right) \sim 1$ [26], which is easy for atoms in an optical trap of crossed configuration.

We thank E. S. Polzik for useful discussions at the 5th Laser Cooling Workshop at Awaji. We thank M. Kitagawa for his interesting lecture and respectful advice. We also acknowledge useful discussions with M. Kozuma and M. Ueda. This work was supported by the Grant-in-Aid for the 21 st century COE, "Center for Diversity and Universality in Physics" from the Ministry of Education, Culture, Sports, Science and Technology (MEXT) of Japan, and Strategic Information Communications R\&D Promotion Programme (SCOPE-S). M. T. is supported by the JSPS.

[1] M. Kitagawa and M. Ueda, Phys. Rev. A 47, 5138 (1993).

[2] Y. Takahashi, M. Fujimoto, T. Yabuzaki, A. D. Singh, M. K. Samal, and B.P. Das, in Proceedings of $C P$
Violation and its Origins, edited by K. Hagiwara (KEK Reports, Tsukuba, 1997).

[3] W. M. Itano, J. C. Bergquist, J. J. Bollinger, J. M. Gilligan, D. J. Heinzen, F. L. Moore, M. G. Raizen, and D. J. Wineland, Phys. Rev. A 47, 3554 (1993).

[4] D. J. Wineland, J. J. Bollinger, W. M. Itano, and D. J. Heinzen, Phys. Rev. A 50, 67 (1994).

[5] B. Julsgaard, A. Kozhekin, and E.S. Polzik, Nature (London) 413, 400 (2001).

[6] M. D. Lukin, S. F. Yelin, and M. Fleischhauer, Phys. Rev. Lett. 84, 4232 (2000).

[7] A. André, L. M. Duan, and M. D. Lukin, Phys. Rev. Lett. 88, 243602 (2002).

[8] A. Kuzmich, N. P. Bigelow, and L. Mandel, Europhys. Lett. 42, 481 (1998).

[9] Y. Takahashi, K. Honda, N. Tanaka, K. Toyoda, K. Ishikawa, and T. Yabuzaki, Phys. Rev. A 60, 4974 (1999).

[10] A. Kuzmich, L. Mandel, J. Janis, Y.E. Young, R. Ejnisman, and N.P. Bigelow, Phys. Rev. A 60, 2346 (1999).

[11] A. Kuzmich, L. Mandel, and N. P. Bigelow, Phys. Rev. Lett. 85, 1594 (2000).

[12] L. K. Thomsen, S. Mancini, and H. M. Wiseman, Phys. Rev. A 65, 061801(R) (2002).

[13] J. M. Geremia, John K. Stockton, and Hideo Mabuchi, Science 304, 270 (2004).

[14] J. Hald, J. L. Sørensen, C. Schori, and E. S. Polzik, Phys. Rev. Lett. 83, 1319 (1999).

[15] C. Schori, B. Julsgaard, J. L. Sørensen, and E. S. Polzik, Phys. Rev. Lett. 89, 057903 (2002).

[16] M. Fleischhauer and M.D. Lukin, Phys. Rev. Lett. 84, 5094 (2000).

[17] M. Fleischhauer and S. Gong, Phys. Rev. Lett. 88, 070404 (2002).

[18] K. Helmerson and L. You, Phys. Rev. Lett. 87, 170402 (2001).

[19] A. Sørensen, L. M. Duan, J. I. Cirac, and P. Zoller, Nature (London) 409, 63 (2001).

[20] L. M. Duan, A. Sørensen, J. I. Cirac, and P. Zoller, Phys. Rev. Lett. 85, 3991 (2000).

[21] A. Sørensen and K. Mølmer, Phys. Rev. Lett. 83, 2274 (1999).

[22] A. S. Sørensen and K. Mølmer, Phys. Rev. A 66, 022314 (2002).

[23] H. Saito and M. Ueda, Phys. Rev. A 68, 043820 (2003).

[24] L. Vernac, M. Pinard, and E. Giacobino, Phys. Rev. A 62, 063812 (2000).

[25] K. Hammerer, K. Mølmer, E. S. Polzik, and J. I. Cirac, Phys. Rev. A 70, 044304 (2004).

[26] L. M. Duan, J. I. Cirac, P. Zoller, and E. S. Polzik, Phys. Rev. Lett. 85, 5643 (2000).

[27] C. Cohen-Tannoudji and J. Dupont-Roc, Phys. Rev. A 5, 968 (1972).

[28] See, for example, Dieter Suter, The Physics of Laser-Atom Interactions (Cambridge University Press, Cambridge, 1997).

[29] K. Honda et al., Phys. Rev. A 66, 021401(R) (2002).

[30] Y. Takasu et al., Phys. Rev. Lett. 90, 023003 (2003).

[31] Y. Takasu et al., Phys. Rev. Lett. 91, 040404 (2003). 